\title{
Complement of Special Relativity and Limitation of General Relativity
}

\author{
KeXin Yao ${ }^{1}$ \\ ${ }^{1}$ Xi' an Institute of Metrology, No.12, Laodong South Road, Xi'an, P. R. China \\ Correspondence: KeXin Yao, Xi'an Institute of Metrology, No.12, Laodong South Road, Xi'an, P. R. China. \\ E-mail: yayydwpq@163.co,029bmsp@163.com
}

$\begin{aligned} & \text { Received: December 17, } 2015 \\ & \text { Accepted: December 24, } 2015 \quad \text { Online Published: January 29, } 2016 \\ & \text { doi:10.5539/apr.v8n1p81 }\end{aligned} \quad$ URL: http://dx.doi.org/10.5539/apr.v8n1p81

\begin{abstract}
For a set of forces being in equilibrium, the equilibrium state will not vary from one observer to another. This generally acknowledged fact has been called as the Force Equilibrium Invariance Axiom. On the basis of this axiom, the force transformation formula can be derived when an object is in motion, so that the force, length, time and mass can be calculated as per the basic transformation formula for the special relativity. According to the results of experimental analysis obtained by J.C. Hafele and R.E. Keating, the concepts of absolute time delay and relative time delay have been put forward herein, so as to solve any problems on the traveling velocity and records of any object being in motion at the real-time. For the properties of the force, the force applied on an object being accelerated will be deemed as the energy transfer force, while the universal gravitation will be deemed as the applied force of field. Both forces have the different basic properties. It is unscientific to consider both forces being the same properties. Based on the Force Equilibrium Invariance Axiom, it is deduced that the gravitational mass can only be the constant irrelevant to the motion of an object. Therefore, the principle of equivalence will not be tenable in theory. Only if the motion velocity of an object is very small relative to the velocity of light, can the principle of equivalence be deemed being approximately tenable. Under this condition, the general relativity can be only consistent with the practical deducing principle. However, if the motion velocity of an object is relatively large, there will be a significant difference between the constant gravitational mass and the inertial mass; as a result, there may be a deviation between the inference on the general relativity and the practical deducing principle. Take the Black Hole as an example. Even if the Black Hole is made almost completely of neutrons, its actual volume is one million times greater than its theoretical volume. Therefore, it is concluded that the Black Hole cannot be deemed as the substance composed of real atoms. It also shows accordingly that, according to the general relativity of theoretical source of Black Hole, the analysis results obtained when an object is in motion at high-velocity can not conform to the reality.
\end{abstract}

Keywords: Force equilibrium, Force transformation, Relative motion, Circular motion, Relative time delay, Absolute time delay, Energy transfer force, Applied force of field, Gravitational mass, Inertial mass, Equivalence principle, Black Hole

\section{Introduction}

There are four fundamental physical quantities in the theory of mechanics, i.e. length $l$, time $t$, mass $m$ and force F. It must be pointed out according to the special relativity that, if the inertial system $Z^{\prime}$ is in motion at the velocity $v$ relative to the inertial system $Z$, it will be observed in $Z$ that, the length $l$ of a stationary object in $Z^{\prime}$ will be translated into $l^{\prime}=l \sqrt{1-v^{2} / c^{2}}$, the time interval $t\left(t_{2}-t_{1}\right)$ into $t^{\prime}=t \sqrt{1-v^{2} / c^{2}}$ and the mass $m$ into $m^{\prime}=m / \sqrt{1-v^{2} / c^{2}}$. However, it isn't specifically and clearly interpreted in the special relativity whether or how to transform the stationary force in $Z^{\prime}$. Therefore, in order to achieve a comprehensive transformation of the fundamental physical quantities in the special relativity, it is necessary to analyze and explore the transformation of force.

Moreover, relative to the rest length, the rest mass is constant; the time is running forward along with the time variation, and always changing its running records; the running velocity of time is determined by the size of time interval $t\left(t_{2}-t_{1}\right)$; if $t$ is large, the time will run quickly; if $t$ is small, the time will run slowly, i.e. the time delay. However, $t^{\prime}=t \sqrt{1-v^{2} / c^{2}}$ is the only transformation formula for an object being in relative motion, where neither the real clock running conditions nor the real clock running records of the stationary object can be determined or reckoned. For example, when $\mathrm{A}$ and $\mathrm{B}$ are in relative motion, if the rest time interval of $\mathrm{B}$ is expressed as $t$, it is judged by $\mathrm{B}$ that $\mathrm{B}$ is in motion at $v$ relative to $\mathrm{A}$, the time interval of $\mathrm{A}$ will be expressed as 
$t^{\prime}=t \sqrt{1-v^{2} / c^{2}}$; however, if the rest time interval of $\mathrm{A}$ is also expressed as $t$ and it is also observed that $\mathrm{B}$ is in motion at $-v$ relative to $\mathrm{A}$, it is judged by $\mathrm{A}$ that the time interval of $\mathrm{B}$ will be expressed as $t^{\prime}=t \sqrt{1-v^{2} / c^{2}}$. Obviously, according to the contradictory judged results of A and B, we cannot determine the real-time running conditions of $\mathrm{A}$ and $\mathrm{B}$ only based on the time transformation formula. How to determine the real-time running records of an object?

It is judged by the general relativity that the force applied on an accelerating object and the universal gravitation will be of the same properties. However, from in-depth analysis, these two forces are fundamentally different in some respects; so, it is unscientific to suppose that both forces be identical. And, as a theoretical basis of general relativity, the equivalence principle is merely deemed as a supposition. Due to lack of the theoretical analysis and convincing experimental proof, the most obvious and fundamental problem is that, when the object is in motion at $v$, whether is the gravitational mass expressed as $m^{\prime}=m \sqrt{1-v^{2} / c^{2}}$ the same as the inertial mass? Obviously, it is critical for the tenable gravitational mass to determine whether the equivalence principle is correct as per this transformation formula. Therefore, it is necessary for general relativity researchers to analyze and solve this critical problem.

\section{Transformation of Force Complemented to the Special Relativity}

\subsection{Force Equilibrium Invariance Axiom and force transformation formula}

After the object $(1 \mathrm{~kg}$ ) has been placed on a spring balance, the pointer of spring balance is set at the $1 \mathrm{~kg}$ scale position, which remains $1 \mathrm{~kg}$ scale not only to someone being stationary relative to the spring balance but also to anyone being in motion relative to the spring balance. This fact shows that a set of forces being in equilibrium in a reference frame can be observed by any other observers in the reference frame. Or rather, a set of forces being in equilibrium will never vary from one observer to another in the reference frame. This is a fact accepted in people's daily life, called as the Force Equilibrium Invariance Axiom. The force transformation formula can be derived on the basis of this Force Equilibrium Invariance Axiom.

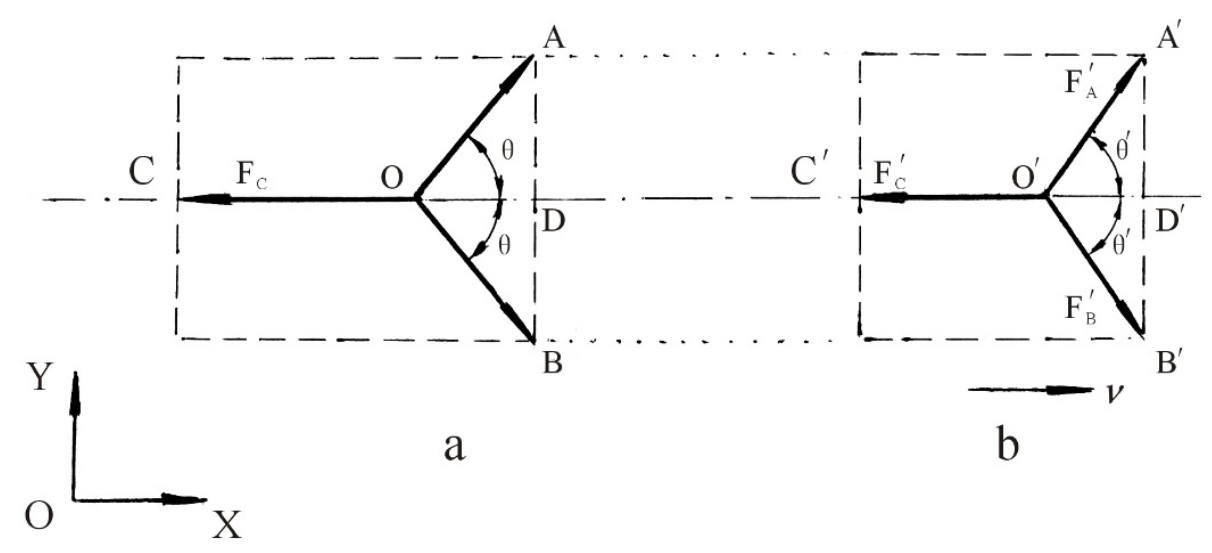

Figure 1. Changes in Size of the Force being in Motion

The three forces $\left(F_{A}, F_{B}\right.$ and $\left.F_{C}\right)$ are in equilibrium (as shown in Figure 1a), including: Applied force of electric field, universal gravitation or spring force. As shown in Figure 1a, the length of OA, OB or OC represents the size of $F_{A}, F_{B}$ or $F_{C}$, respectively; $F_{C}$ is parallel to the $\mathrm{X}$ axis; $F_{A}$ is equivalent to $F_{B}$; the included angle between $F_{A}$ or $F_{B}$ and the $\mathrm{X}$ axis is $\theta$ (irrespective of the positive or negative angle of $\theta$ ). Since the three forces are in equilibrium, it also can be referred to as $F_{A} \cos \theta+F_{B} \cos \theta=F_{C}$. Since $F_{A}$ is equivalent to $F_{B}$, for simple derivation, if $F_{A}=F_{B}=F$, it also can be referred to as $2 F \cos \theta=F_{C}$.

The three forces (as shown in Figure 1a) are in motion at $v$ parallel to the $\mathrm{X}$ axis (as shown in Figure 1b). According to the special relativity, the length along the $v$ direction (X direction) will be shortened; $F_{C}$ (as shown in Figure $1 b$ ) will be shortened as $F_{C}^{\prime} ; F_{A}$ and $F_{B}$ will be shortened as $F_{A}^{\prime}$ and $F_{B}^{\prime}$ respectively. According to the Force Equilibrium Invariance Axiom, $F_{C}^{\prime}, F_{A}^{\prime}$ and $F_{B}^{\prime}$ are still in the state of equilibrium; $F_{A}^{\prime}$ is naturally equivalent to $F_{B}^{\prime}$; if $F^{\prime}=F_{A}^{\prime}=F_{B}^{\prime}$, it also can be referred to as $2 F^{\prime} \cos \theta^{\prime}=F_{C}^{\prime}$.

The relation between $F_{C}$ and $F_{C}^{\prime}$ can be inferred according to any changes in applied force of electric field being in motion. 


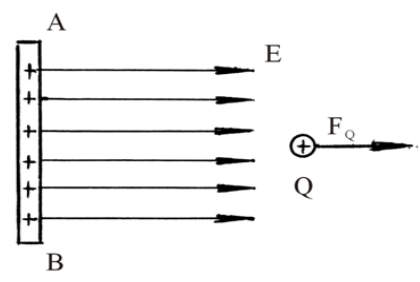

a

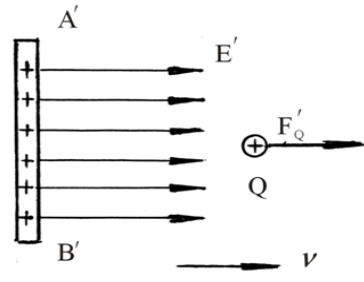

b

Figure 2. Electric Field being in Motion Parallel to the Intensity Line of Electric Field $E$

The electric charge $\mathrm{Q}$ is in a uniform electric field generated by the "infinitely great" charged plate (as shown in Figure 2a). $E$ represents the intensity of electric field; $A B$ represents a certain cross-section of this charged plate; obviously, the applied force of $Q$ will be expressed as $F_{Q}=E_{Q}$. As shown in Figure $2 \mathrm{~b}$, after $A B$ is in motion at the velocity $v$ parallel to E, $A B$ has been transformed as $A^{\prime} B^{\prime}$. According to the special relativity, after it is in motion along the length vertical to the direction of $v$, the size of length will be invariable; it also can be referred to as $A^{\prime} B^{\prime}=A B$, naturally resulting in the intensity of electric field of $A^{\prime} B^{\prime}: E^{\prime}=E$. Therefore, when $A^{\prime} B^{\prime}$ is in the electric field, the applied force of $Q$ will be expressed as: $F_{Q^{\prime}}=E^{\prime} Q=E Q=F_{Q}$. It follows from this fact that, after the force $F_{Q}$ is in motion along the direction parallel to $F_{Q}$, the size of force will be invariable $\left(F_{Q}=F_{Q}\right)$. For the force (as shown in Figure 1), it also can be referred to as $F_{C}^{\prime}=F_{C}$. Since $F_{C}^{\prime}=2 F^{\prime} \cos \theta^{\prime}$ and $F_{C}=2 F \cos \theta$, it also can be referred to as $F^{\prime} \cos \theta^{\prime}=F \cos \theta$.

$$
F^{\prime}=F \frac{\cos \theta}{\cos \theta^{\prime}}
$$

As can be seen from Figure 1:

$$
\cos \theta=\frac{O D}{\sqrt{O D^{2}+A D}} \quad \cos \theta^{\prime}=\frac{O^{\prime} D^{\prime}}{\sqrt{O^{\prime} D^{\prime 2}+A^{\prime} D^{\prime 2}}}
$$

On account of the same length being vertical to the direction of $v\left(A^{\prime} D^{\prime}=A D\right)$, according to the special relativity, the length $O^{\prime} D^{\prime}$ along the direction of $v$ can be calculated as per the length transformation formula $\left(O^{\prime} D^{\prime}=O D \sqrt{1-v^{2} / c^{2}}\right)$. According to the equation $A^{\prime} D^{\prime}$ and $O^{\prime} D^{\prime}$, the $\cos \theta^{\prime}$ can be transformed as:

$$
\begin{aligned}
\cos \theta^{\prime} & =\frac{O D \sqrt{1-v^{2} / c^{2}}}{\sqrt{O D^{2}\left(1-v^{2} / c^{2}\right)+A D^{2}}} \\
F^{\prime} & =F \frac{\cos \theta}{\cos \theta^{\prime}}=F \frac{O D}{\sqrt{O D^{2}+A D^{2}}} \cdot \frac{\sqrt{O D^{2}\left(1-v^{2} / c^{2}\right)+A D^{2}}}{O D \sqrt{1-v^{2} / c^{2}}} \\
& =F \sqrt{\frac{\left(O D^{2}+A D^{2}\right)-O D^{2} v^{2} / c^{2}}{\left(O D^{2}+A D^{2}\right)\left(1-v^{2} / c^{2}\right)}}
\end{aligned}
$$

After $\cos \theta^{\prime}=O D / \sqrt{O D^{2}+A D^{2}}$ is substituted into the above equation, it also can be referred to as:

$$
F^{\prime}=F \sqrt{\frac{1-\cos ^{2} \theta v^{2} / c^{2}}{1-v^{2} / c^{2}}}
$$

Since $F^{\prime}=F \cos \theta / \cos \theta^{\prime}$ and $\cos \theta^{\prime}=F \cos \theta / F^{\prime}$, it also can be referred to as:

$$
\begin{aligned}
\cos \theta^{\prime} & =\cos \theta \sqrt{\frac{1-v^{2} / c^{2}}{1-\cos ^{2} \theta v^{2} / c^{2}}} \\
\theta^{\prime} & =\cos ^{-1} \cos \theta \sqrt{\frac{1-v^{2} / c^{2}}{1-\cos ^{2} \theta v^{2} / c^{2}}}
\end{aligned}
$$


The above equations (1) and (2) will be deemed as the transformation formula when the force is in motion at $V$. The force transformation formula is the same as the length, time and mass transformation formula, i.e. the fundamental transformation formula for special relativity.

2.2 Electric Field Distribution of Charged Particles Being in Motion as derived from the Force Transformation Formula

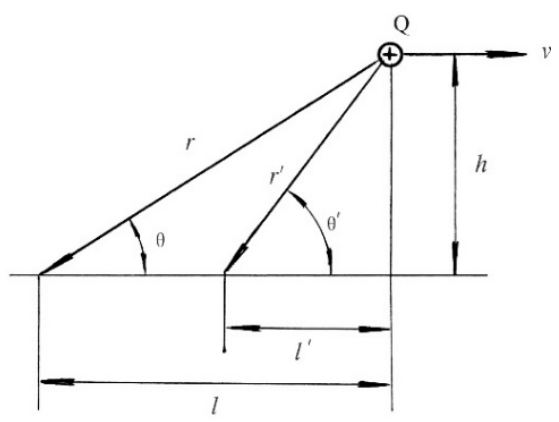

Figure 3. $\mathrm{r}$ will be transformed as $\mathrm{r}^{\prime}$ when the charged particle is in motion at $V$.

The positively charged particle $\mathrm{Q}$ is in motion at $v$ (as shown in Figure 3). The rest length $l$ (as shown in Figure 3) will be shortened as $l^{\prime}=l \sqrt{1-v^{2} / c^{2}}$ after motion. The size of height being vertical to the direction of $v$ will be invariant before and after motion. $\mathrm{r}$ (as shown in Figure 3) will be shortened as $\mathrm{r}^{\prime}$ after motion. It can be seen from Figure 3 that if $l=r \cos \theta, l^{\prime}=l \sqrt{1-v^{2} / c^{2}}=r \cos \sqrt{1-v^{2} / c^{2}}$ and $h=r \sin \theta$, it also can be referred to as:

$$
\begin{aligned}
r^{\prime} & =\sqrt{l^{\prime 2}+h^{2}}=\sqrt{r^{2} \cos ^{2} \theta\left(1-v^{2} / c^{2}\right)+r^{2} \sin ^{2} \theta} \\
& =r \sqrt{1-\cos ^{2} \theta v^{2} / c^{2}}
\end{aligned}
$$

Since $h=r \sin \theta=r^{\prime} \sin \theta^{\prime}$, it can be referred to as $\sin \theta^{\prime}=\sin \theta r / r^{\prime}$. After the equation (3) is substituted into the above equation, it also can be referred to as:

$$
\sin \theta^{\prime}=\sin \theta / \sqrt{1-\cos ^{2} \theta v^{2} / c^{2}}
$$

Since $\mathrm{Q}$ is the electric field (the intensity of electric field $\mathrm{E}$ ), the applied force will be referred to as $\mathrm{F}=\mathrm{EQ}$, i.e. the intensity of electric field $\mathrm{E}$ is proportional to the applied force F; so, the force transformation formula also can be referred to as the transformation formula for the intensity of electric field as follows:

$$
E^{\prime}=E \sqrt{\frac{1-\cos ^{2} \theta v^{2} / c^{2}}{1-v^{2} / c^{2}}}
$$

The equation (5) can be transformed as:

$$
\begin{aligned}
E^{\prime} & =E \sqrt{\frac{1-\cos ^{2} \theta v^{2} / c^{2}}{1-v^{2} / c^{2}}} \cdot \frac{\left(1-\cos ^{2} \theta v^{2} / c^{2}\right)\left(1-v^{2} / c^{2}\right)}{\left(1-\cos ^{2} \theta v^{2} / c^{2}\right)\left(1-v^{2} / c^{2}\right)} E \\
& =\frac{\left(1-\cos ^{2} \theta v^{2} / c^{2}\right)^{3 / 2}}{\left(1-v^{2} / c^{2}\right)^{3 / 2}} \cdot \frac{1-v^{2} / c^{2}}{1-\cos ^{2} \theta v^{2} / c^{2}} E \\
& =\frac{1-v^{2} / c^{2}}{\left(\frac{1-v^{2} / c^{2}}{1-\cos ^{2} \theta v^{2} / c^{2}}\right)^{3 / 2}} \cdot \frac{E}{1-\cos ^{2} \theta v^{2} / c^{2}}
\end{aligned}
$$


Since $1-v^{2} / c^{2}=1-\left(\sin ^{2} \theta+\cos ^{2} \theta\right) v^{2} / c^{2}$, the above equation can be transformed as:

$$
E^{\prime}=\frac{1-v^{2} / c^{2}}{\left(1-\frac{\sin ^{2} \theta v^{2} / c^{2}}{1-\cos ^{2} \theta v^{2} / c^{2}}\right)^{3 / 2}} \cdot \frac{E}{1-\cos ^{2} \theta v^{2} / c^{2}}
$$

After equations (3) $\sqrt{1-\cos ^{2} \theta v^{2} / c^{2}}=r^{\prime} / r$ and (4) $\sin \theta^{\prime}=\sin \theta / \sqrt{1-\cos ^{2} \theta v^{2} / c^{2}}$ are substituted into the above equation, it also can be referred to as:

$$
E^{\prime}=\frac{1-v^{2} / c^{2}}{\left(1-\sin ^{2} \theta^{\prime} v^{2} / c^{2}\right)^{3 / 2}} \cdot \frac{r^{2}}{r^{\prime 2}} E
$$

Since $E=k Q / r^{2}$, after it is substituted into the above equation, it also can be referred to as:

$$
E^{\prime}=K \frac{1-v^{2} / c^{2}}{\left(1-\sin ^{2} \theta^{\prime} v^{2} / c^{2}\right)^{3 / 2}} \cdot \frac{Q}{r^{\prime 2}}
$$

The equation (6) will be deemed as the electric field distribution formula for charged particle being in motion at $v$. By comparison, this equation will be identical to the formula derived by electrodynamics.

When the force-applied object is in motion, the force will be transformed like as the length, time and mass. For example, when the charged particle iss are in motion in a magnetic field, the Lorentz force applied on the particles will be transformed. For the specific analysis, see Reference 4.

\section{Absolute Time Delay and Relative Time Delay Complemented to the Special Relativity}

\subsection{Two Types of Object Motion}

In our daily life, we can observe two types of object motion. The first one is relative motion in which either side can observe the opposite side in motion. For example, we can see from the train window that an opposite train is traveling, which might be traveling or stopping, while our train is traveling as a matter of fact. This is relative motion. According to the special relativity, each side being in relative motion may consider that the time interval of the opposite side being in motion is shortened, i.e. the time delay. The second type of motion is circular motion, different from relative motion. For example, the ferris wheel cabin of playground is rotating around the center of ferris wheel, or the man-made satellite or the Moon is rotating around the Earth. Unlike the relative motion, the circular motion refers to a universally acknowledged motion in which the circular object can revolve around the center of a circle rather than the motion in which the circular object and the center of a circle can revolve around each other. Taking the ferris wheel as an example, the center of ferris wheel and the person on the stationary ground relative to the center of ferris wheel can consider that the ferris wheel cabin is rotating around the center of ferris wheel, while the person in ferris wheel cabin can also consider that he/she is rotating around the center of ferris wheel, i.e. the circular motion of ferris wheel cabin will be deemed as an accepted motion rather than a relative motion. Similarly, the motion of the man-made satellite or the Moon revolving around the Earth will be deemed as an accepted motion as well.

The accepted velocity of circular motion can be defined as the absolute velocity. Naturally, the velocity of relative motion can be defined as the relative velocity.

If the rotational velocity of an object being in circular motion is set as $\mathrm{v}$, and the time interval of the center of rotation is set as $\mathrm{T}$, then according to the special relativity, the time interval of the object being in circular motion will be inevitably expressed as:

$$
t^{\prime}=t \sqrt{1-v^{2} / c^{2}}
$$

Obviously, here $t^{\prime}$ represents the time interval between the object being in circular motion and the center of circle. Such $t^{\prime}$ is not relative, but absolute. Relative to $t, t^{\prime}$ is reduced. A reduced $t^{\prime}$ indicates that the time motion velocity is low, i.e. time delay. Such accepted time delay $t^{\prime}$ will be defined as absolute time delay, e.g. time delay of the Earth relative to the Sun, time delay of the Moon relative to the Earth, etc. 
Next, when it comes to the time motion condition, we will only use the term "time interval"; when the time interval is relatively reduced, it will be referred to as time delay.

There are a variety of circular motions in the universe; each has its own different absolute velocity and absolute time delay. How to compare with their sizes of motion? First of all, there must be a standard to make comparison. For us, of course, the time interval t of the rotation center of the Earth (i.e. the Earth's south and north poles, say 1 hour) will prevail.

Relative to $t$, the time interval $t_{\mathrm{m}}$ of the Moon is reduced, i.e. $t_{\mathrm{m}}$ in relation to $t$ is time delay. Assuming that the Moon moves around the Earth at a velocity of $\mathrm{v}_{\mathrm{m}}$, then the time interval of the Moon will be expressed as:

$$
t_{m}=t \sqrt{1-v_{\mathrm{m}}^{2} / c^{2}}
$$

If the Earth moves around the Sun at $\mathrm{v}$, and the time interval of the Sun is expressed as $t_{0}$, then it can be derived that $t=t_{0} \sqrt{1-v^{2} / c^{2}}$, namely,

$$
t_{0}=t / \sqrt{1-v^{2} / c^{2}}
$$

Obviously, $t_{0}$ is greater than $\mathrm{t}$, so $t_{0}$ cannot be referred to as time delay. If a certain planet of the Sun moves around it at $\mathrm{v}_{\mathrm{x}}$, the time interval of $\mathrm{x}$ planet will be expressed as:

$$
t_{\mathrm{x}}=t_{0} \sqrt{1-v_{x}^{2} / c^{2}}=t \sqrt{1-v_{x}^{2} / c^{2}} / \sqrt{1-v^{2} / c^{2}}
$$

It can be observed that, if $\mathrm{v}_{\mathrm{x}}>v$, then $t_{\mathrm{x}}<t$. Relative to $\mathrm{t}, \mathrm{t}_{\mathrm{x}}$ will be defined as time delay; on the contrary, the time speeds up.

If a certain satellite moves around the Moon at $V_{\mathrm{s}}$, then the time delay of the Moon's satellite will be expressed as:

$$
t_{\mathrm{s}}=t_{m} \sqrt{1-v_{s}^{2} / c^{2}}=t \sqrt{1-v_{s}^{2} / c^{2}} \sqrt{1-v^{2} / c^{2}}
$$

The comparison between time intervals of other objects being in circular motion can be analogized according to the above calculation methods.

Each celestial body in the universe has its own different time interval, and each generally rotates by itself. Relative to the axis of rotation, the celestial body has its different velocity of rotation at different locations; there is also a difference between time intervals. In the case of the Earth, the equatorial radius of the Earth is about $6,378 \mathrm{~km}$; it takes 24 hours for the Earth to rotate around its axis once; the velocity of rotation of the Earth's land surface can be calculated as $0.464 \mathrm{~km} / \mathrm{s}$. Along with an increase in terrestrial latitude, the velocity of rotation of different points on the Earth's land surface will decrease. If the terrestrial latitude somewhere on the Earth is expressed as $\theta$, then the velocity of rotation of this point relative to the axis here will be expressed as $v_{\theta}=0.464 \cos \theta \mathrm{km} / \mathrm{s}$ and the time interval at this point will be expressed as:

$$
t_{\theta}=t \sqrt{1-0.2153 \cos ^{2} \theta / c^{2}}
$$

It is concluded from the above analysis that any celestial body or its different location has its own fixed time interval. The locations with the same time interval can be divided into equal time interval zones. For example, the Earth's surface with the same latitude can be divided into equal time interval zones. If a certain object is placed at $t_{1}$ time zone, the time motion velocity of such object will operate as per $t_{1}$; if such object is placed at $t_{2}$ time zone, its time motion velocity will operate as per $\mathrm{t}_{1}$, and so on.

\subsection{Experiment Conducted by $J \cdot C \cdot$ Hafele and $R \cdot E \cdot$ Keating}

In 1971, J.C.Hafele and R.E.Keating carried out an experiment for the relationship between time delay and motion velocity. They placed four caesium atomic clocks on an aircraft stopping near the equator. After the aircraft traveled along the equator from east to west so as to make a complete cycle around the Earth, it was found that the average reading of the four caesium atomic clocks was $273 \times 10^{-9}$ seconds faster than that of the caesium atomic clock placed on the ground (surface phenomenon was negative time delay). However, after the aircraft traveled along the equator from west to east so as to make a complete cycle around the Earth, it was found that the average reading of the four caesium atomic clocks was $59 \times 10^{-9}$ seconds slower than that of the caesium atomic clock placed on the ground (Reference 1). Why could such a result occur? As previously 
described, the absolute time delay of an object or any point on the ground relative to the Earth's axis will depend on its motion velocity relative to the Earth's axis. When both the aircraft and equatorial ground are rotating around the Earth's axis, the velocity of rotation of equatorial ground around the Earth's axis will be set as $v_{1}$; the flight velocity of the aircraft will be set as $v$. When the aircraft is flying to the west, the motion direction of the aircraft will be opposite to the direction of the Earth's rotation and the actual velocity of rotation of the aircraft around the Earth's axis will be set as $v_{2}=v_{1}-v$. When the aircraft is flying to the east, the motion direction of the aircraft is consistent with the direction of the Earth's rotation and the actual velocity of rotation of the aircraft around the Earth's axis will be set as $v_{3}=v_{1}+v$. It can thus be seen that the time interval of equatorial ground relative to the Earth's axis (time interval T) can be expressed as $t_{1}=t \sqrt{1-v_{1}^{2} / c^{2}}$; the time interval of aircraft to the west relative to the Earth's axis can be expressed as $t_{2}=t \sqrt{1-\left(v_{1}-v\right)^{2} / c^{2}}$; and the time interval of the aircraft to the east relative to the Earth's axis can be expressed as $t_{3}=t \sqrt{1-\left(v_{1}+v\right)^{2} / c^{2}}$. Obviously, if $t_{2}>t_{1}$, namely, the time motion velocity of the aircraft to the west is faster in relation to the Earth's axis, the absolute time delay of the aircraft to the west is less than the absolute time delay of equatorial ground ( $t_{2}$ is large; time delay is small); if $t_{3}<t_{1}$, the absolute time delay of the aircraft to the east is greater than the absolute time delay

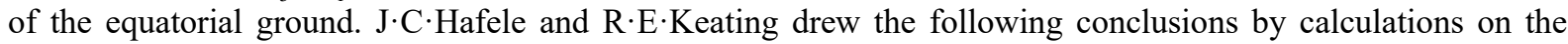
basis of such difference: The caesium atomic clock on the aircraft to the west would be $275 \times 10^{-9}$ faster than the caesium atomic clock on equatorial ground, consistent with the measured readings. In addition, they reckoned from $t_{3}<t_{1}$ that the caesium atomic clock on the aircraft to the east would be $40 \times 10^{-9}$ slower than the caesium atomic clock on the equatorial ground. Outwardly, although there is a major difference between this result and the measured readings $\left(59 \times 10^{-9}\right.$ seconds), this is only a comparison difference, rather than a calculation error. For example, if the height of a wall is up to $300 \mathrm{~cm}$ and the height of a tree is up to $301 \mathrm{~cm}$, then the tree is $1 \mathrm{~cm}$ higher than the wall. However, if the calculated height of the tree is up to $304 \mathrm{~cm}$, then the tree is $4 \mathrm{~cm}$ higher than the wall according to calculations. Obviously, there is a major difference between 4 and 1; however, this difference can be deemed as a comparison difference rather than a calculation error. The calculation error will be deemed as the error between 304 and 301, i.e. the error is less than one percent (1\%).

In accordance with our requirements, one hour of the equatorial caesium atomic clock, i.e. 3,600 seconds, is set as the standard time interval $t_{1}$. When the equatorial caesium atomic clock has gone forward by $50 t_{1}$ (i.e. $1.8 \times 10^{5}$ seconds) after the aircraft completes one circle around the Earth, the measured motion time of the caesium atomic clock on the aircraft to the east will be $50 \mathrm{t}_{3}=1.8 \times 10^{5} \mathrm{~s}-59 \times 10^{-9} \mathrm{~s}$, but the theoretically calculated motion time of the caesium atomic clock is $50 t_{3}^{\prime}=1.8 \times 10^{5} \mathrm{~s}-40 \times 10^{-9} \mathrm{~s}$. The ratio between the calculated value and the measured value $\left(t_{3}^{\prime} / t_{3}\right)$ can be expressed as $\left(1.8 \times 10^{5}-40 \times 10^{-9}\right) /\left(1.8 \times 10^{5}-59 \times 10^{-9}\right)$. Thus it can be seen that there is a relatively small error between the theoretically calculated value $t_{3}^{\prime}$ and the measured value. Therefore, we may consider that the analysis results are consistent with the experimental results obtained by $\mathrm{J} \cdot \mathrm{C} \cdot$ Hafele and R.E. Keating.

The experimental results obtained by $\mathrm{J} \cdot \mathrm{C} \cdot \mathrm{Hafele}$ and $\mathrm{R} \cdot \mathrm{E} \cdot \mathrm{Keating}$ have proved that the velocity of rotation of the equatorial ground, the aircraft to the west or the aircraft to the east around the Earth's axis could be referred to as absolute velocity; They also demonstrated that the circular motion velocity could be referred to as absolute velocity andthe circular motion time delay as absolute time delay. Moreover, it shows the appropriateness of the method for determining the time interval as per the division of time zones.

\subsection{Relative Time Delay and Absolute Time Delay \& Predication of Real-Time Progress}

In the experiments conducted by J.C. Hafele and R.E. Keating, the flight velocity of the aircraft flying to the east is the same as to the west, and people on the equatorial ground are supposed to observe that the time delay results of the aircraft to the east can be identical with those of the aircraft flying to the west. However, the experimental results have shown that the time delay results of the aircraft flying to the east are different from those of the aircraft to the west; especially the time of the aircraft to the west, instead of being delayed, speeds up. It is proved beyond doubt that the relative time delay judged by the people as per the relative velocity is fundamentally different from the real absolute time delay of a moving object. The experimental results obtained by J.C. Hafele and R.E. Keating affirmed the correctness of the method for calculating the absolute time delay as per the circular motion velocity. Moreover, the muon lifetime experiments have also confirmed that the relative time delay could be deemed as a real observation. How to understand the two seemingly different but truthful judgments?

In connection with any changes in the length of an object, we may understand the above-mentioned two different judgments. As we know, when an object is in motion at a velocity $v$, the length $l$ of the object along the direction of motion will be shortened as $l^{\prime}=l / \sqrt{1-v^{2} / c^{2}}$. We observe that $l^{\prime}$ of the object being in motion is real, but the length $l$ of the object is also real without any changes, i.e. both $l$ and $l^{\prime}$ are real. Observers using 
different reference systems can obtain such an observation. Changes in time are similar to changes in length. That there is distinction between relative time delay and absolute time delay with respect to time could also be observed by observers using different reference systems.

Changes in length and time are related to the motion velocity observed by the observer. The observer on the equatorial ground being in motion at a velocity $v_{1}$ observes that the relative velocity of the aircraft flying to the west or the aircraft to the east relative to him can be expressed as $v$; both the length contraction and time delay of the two aircrafts are naturally identical. However, an observer not standing on the equatorial ground will not observe that the relative velocity of the aircraft flying to the west is identical with the relative velocity of the aircraft flying to the east relative to him. For example, an observer on the Earth's north or south pole observes that the relative velocity of the aircraft flying to the west relative to him is expressed as $v_{1}-v$ while the relative velocity of the aircraft flying to the east relative to him is expressed as $v_{1}+v$; both the length contraction and time delay of the two aircrafts are significantly different. That is because observers using different reference systems will observe that the same object will be provided with different length contractions and relative time delay, which, though different, will be deemed as the real observation.

Under normal circumstances, the relative time delay is different from the absolute time delay. However, if an observer is located at the center of a circle of the object being in circular motion or within the stationary area relative to this center of a circle, i.e. the area where the absolute motion of the object has been accepted as mentioned before, then the observer will observe the relative velocity of the object being in circular motion relative to him, i.e. the absolute velocity of the object being in circular motion, and the relative time delay of the object will be referred to as the absolute time delay. For example, the relative time delay of the particle being in circular motion in synchrocyclotron as observed by us will be referred to as the real absolute time delay of the particle.

The experimental results obtained by J.C. Hafele and R.E. Keating show that when a stationary object undergoes a motion process before returning back to rest, the rest length and rest mass of the object will not change, but the time progress of the moving object is different from the time progress of a non-moving object. (When the aircraft returns to the ground, the time progress of the aircraft's caesium atomic clock is different from the time progress of the ground caesium atomic clock). Also, such changes in time progress will be related to the motion history of an object, e.g. the aircraft's flight direction, velocity, flight time, etc.

Based on the foregoing analyses, we can easily calculate the corresponding time process with respect to different motion histories of an object. For example, according to the time standard of one hour (as expressed in h) relative to the Earth's axis, we can leave a clock at the terrestrial latitude $\left(45^{\circ}\right)$ on the Earth for $\mathrm{n}_{1} \mathrm{~h}$, then on the Moon for $\mathrm{n}_{2} \mathrm{~h}$ and then on the Sun's x planet for $\mathrm{n}_{3} \mathrm{~h}$, and then return it to the ground. According to the above motion history, we can calculate the cumulative absolute time process of this clock as per the following formula:

$$
\left(n_{1} \sqrt{1-0.2153 / 2 c^{2}}+n_{2} \sqrt{1-v_{m}^{2} / c^{2}}+n_{3} \sqrt{1-v_{x}^{2} / c^{2}} / \sqrt{1-v^{2} / c^{2}}\right) h
$$

We can give a very simple and clear explanation of the twin paradox problem. For example, twins A and B can be placed at two locations with an absolute time interval of $t_{A}$ and $t_{\mathrm{B}}$; if $t_{A}$ is less than $t_{\mathrm{B}}$, i.e. the time motion velocity of time zone $t_{A}$ is slow, when $\mathrm{A}$ and $\mathrm{B}$ meet each other at one location after a period of time, $\mathrm{A}$ will inevitably become younger than $\mathrm{B}$; on the contrary, if $t_{A}$ is greater than $t_{\mathrm{B}}, \mathrm{B}$ will inevitably become younger than A. Take the experiment conducted by J.C. Hafele and R.E. Keating as an example. Suppose B is located on the equatorial ground, when A travels by aircraft to the east around the Earth and then returns to the ground to meet B after a period of time, A will inevitably become younger than B; however, when A travels by aircraft to the west around the Earth and then returns to the ground to meet B after a period of time, B will inevitably become younger than A.

\section{Applicable Scope and Limitation of General Relativity}

\subsection{Problems on General Relativity}

According to the general relativity, the force applied on an object being accelerated will be equivalent to the universal gravitation. However, as a result of analysis, the force applied on an object being accelerated will be deemed as the energy transfer applied force. For example, Object A can transfer its energy to Object B (mass $m$ ). $\mathrm{B}$ moves for a distance $\mathrm{S}$ under the acting force $\mathrm{F}$ of $\mathrm{A}$ at the time $\mathrm{t}$; the output energy of $\mathrm{A}$ will be expressed as FS. Since B moves under the acting force $\mathrm{F}$ at the acceleration of $a$, if $S=a t^{2} / 2$, it will be also referred to as $F S=F a t^{2} / 2$. B has obtained the velocity $v=a t$ under the acting force $\mathrm{F}$ at the time $\mathrm{t}$; the energy obtained by 
B will be also referred to as $m v^{2} / 2=m a^{2} t^{2} / 2$. The output energy of A will be equivalent to the input energy of $\mathrm{B}$; so the following formula can be obtained:

$$
\frac{F a t^{2}}{2}=\frac{m a^{2} t^{2}}{2}
$$

This equation will be deemed as the formulation of accelerating acting force: $F=m a$; that is to say, $F=m a$ refers to the applied force of A for which B can obtain the energy. As can be seen that as long as there is the energy transfer force to exist, it will be accompanied by energy transfer process. The universal gravitation will be deemed as the applied force of field. There is no any relationship between the applied force of field and the energy transmission. Even if an object being in motion can obtain the kinetic energy under the universal gravitation, this kinetic energy can be resulted from the potential energy conversion of the object, rather than the gravitational output energy.

In addition, the universal gravitation will be deemed as the applied force of field. The field will be mainly characterized by the constant field flux of any surface, i.e. there is no any correlation between the field flux and the motion of the observer, while the size of $m$ of energy transfer force $F=m a$ is related to the motion velocity, i.e. $m^{\prime}=m / \sqrt{1-v^{2} / c^{2}}$. If the energy transfer force is equivalent to the universal gravitation, the observer being in motion at $v$ can observe that $m$ generating the gravitational field will be increased to $m^{\prime}=m / \sqrt{1-v^{2} / c^{2}}$, while the field flux of any one surface will be increased to $1 / \sqrt{1-v^{2} / c^{2}}$ accordingly. This judgment is clearly inconsistent with the basic properties of the field; it also means that the energy transfer force is not possibly equivalent to the universal gravitation.

Further, the energy transfer force is generally constant and the acceleration is also constant accordingly. However, the universal gravitation is generally inversely proportional to the square of the distance between gravitational objects; the acceleration generated by the object in the gravitational field is naturally inversely proportional to the square of the distance between gravitational objects; namely, the acceleration being in motion in the gravitational field will never be constant, and there is a fundamental difference between the energy transfer force and the universal gravitation.

Most importantly, the equivalence principle is the basis of general relativity. It is supposed according to the equivalence principle that the gravitational mass is equivalent to the inertial mass. If this supposition is tenable, the observer being in motion at a relatively high velocity $v$ relative to the Sun will observe that the Sun and its planets are in motion at the velocity $-v$ (the rotating velocity $v$ of planets around the Sun is relatively small and can be ignored); their gravitational mass can be increased $1 / \sqrt{1-v^{2} / c^{2}}$ times. Thus, the universal gravitation between the Sun and its planets can be inevitably increased $1 /\left(1-v^{2} / c^{2}\right)$ times. As a result, the universal gravitation of the Sun on its planets will be greater than the centrifugal force of the Sun's planets on the Sun; the planets are absorbed by the Sun; all the planets have disappeared; the solar system has become the lonely Sun. Obviously, the observer being in motion at $v$ cannot see this result. Therefore, the gravitational mass is unlikely equal to the inertial mass; the equivalence principle will not be theoretically tenable; and there is naturally also a problem on the general relativity.

\subsection{Gravitational Mass of an Object Referring to the Constant Unrelated to the Object Motion}

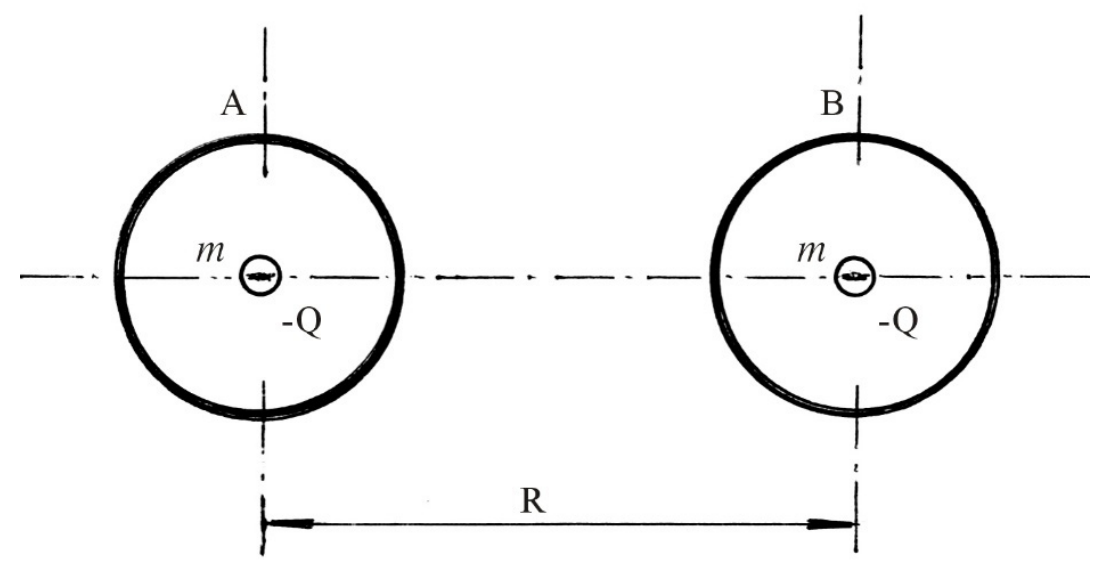

Figure 4. Gravitational Mass Unrelated to the Object Motion 
Object $\mathrm{A}$ and Object $\mathrm{B}$ have the same mass $m$ and carry the equivalent negative charge -Q (as shown in Figure 4), and the distance between Object A and B will be expressed as R. If the mutual universal gravitation between $\mathrm{A}$ and $\mathrm{B}$ is exactly equivalent to the mutual repulsion generated by the negative charge - $\mathrm{Q}$ contained in $\mathrm{A}$ and $\mathrm{B}$, the universal gravitation and repulsion between $\mathrm{A}$ and $\mathrm{B}$ will be in equilibrium, and the distance $\mathrm{R}$ between $\mathrm{A}$ and $\mathrm{B}$ will be invariable and constant.

According to the Force Equilibrium Invariance Axiom, an observer being in motion at any velocity can observe that $\mathrm{A}$ and $\mathrm{B}$ will also inevitably be in equilibrium. It is unlike for the observer to observe that $\mathrm{A}$ and $\mathrm{B}$ will come into collision with each other or gradually separate from each other. It is only possible to meet this condition that the observer can also observe the universal gravitation between $\mathrm{A}$ and $\mathrm{B}$ equivalent to the electrostatic repulsion between A and B. Thus, we can inevitably draw the following conclusion that the gravitational mass is equivalent to the carried charge, i.e. the constant being independent of the motion.

\subsection{General Relativity Applying to an Object Being in Motion at the Low Velocity}

The gravitational mass will be deemed as the constant unrelated to the motion, while the inertial mass will be related to the velocity of motion. This means that, the gravitational mass and the inertial mass will be deemed as the physical quantities being of the different properties; the gravitational mass is not possibly equivalent to the inertial mass. However, if the motion velocity of an object is less than the velocity of light, e.g. the motion velocities of the Sun's planets, man-made satellites and other planets are less than $1 / 6000$ of the velocity of light, then there is a very small difference the inertial mass of an object and the rest mass of an object; it can be deemed that the inertial mass of an object is equivalent to the rest mass of an object, i.e. the inertial mass can be approximately regarded as the constant; under this condition, there is a fixed proportional relation between the inertial mass and the rest mass; through a transformation coefficient, it can be deemed that the gravitational mass is equivalent to the inertial mass; like this, through a transformation coefficient, it can be deemed that the length (height of water column) is equivalent to the pressure; under this condition, the equivalence principle will be tenable; the inference made according to the general relativity will be in line with the reality; for example, the inference on the perihelion precession of the Mercury, GPS positioning and other items as made according to the general relativity will be consistent with the reality. However, if the motion velocity $v$ of an object is relatively high, the inertial mass $m^{\prime}=m \sqrt{1-v^{2} / c^{2}}$ is significantly greater than the gravitational mass; the equivalence principle will not be tenable; the inference made according to the general relativity will be naturally divorced from the reality. For example, it is not correct to judge according to the general relativity that Black Hole exists when an object is in motion at the high velocity. Obviously, if the gravitational mass is supposed to be equivalent to the inertial mass and an object is in motion at the high velocity, the gravitational mass will become large as the inertial mass and the velocity of an object come close to the velocity of light and the gravitational mass will become very large, resulting in the Black Hole thereby. If the gravitational mass is constant and invariant, the Black Hole will never exist naturally.

It can also be judged from the aspect of the material structure that the Black Hole will not exist.

According to the Black Hole theory, for a black-hole sphere with a diameter of $120 \mathrm{~m}$, its mass is equivalent to the total mass of four suns, i.e. the total mass of 1.3 million earths and the Earth's volume is $10^{15}$ times greater than that of the black-hole sphere with a diameter of $120 \mathrm{~m}$; in other words, the density of black-hole matter is $10^{21}$ times greater than that of the Earth, which means the mass of a black-hole matter about the same size as a grain of rice can be 1000 times greater than the total mass of all persons on the Earth (taking the average mass of a person at $50 \mathrm{~kg}$ ). It is well-known that all the matters in the universe consist of several elements among the 118 kinds of elements known. So far, no other cosmic matters have been found to make an exception yet.

However, according to calculations based on the density of black-hole matter, the atomic size of black-hole matter is equivalent to only $1 / 10^{21}$ of the atomic size of matter on the Earth. This is clearly impossible. The reason is that, if we assume the Black Hole is also composed of atoms, after all atoms of the Black Hole have collapsed, all the electrons around such atoms will fall on protons and change them into neutrons, and these neutrons will be gathered together to become a large-sized neutron, then the volume of such a large-sized neutron will be one million times greater than the theoretical volume of the Black Hole. Obviously, there is no possibility of such atoms in reality. Thus, it can be concluded that it is impossible for the Black Hole to be composed of real atoms; that is to say it is impossible for the Black Hole to exist as a real matter.

The general relativity can be deemed as the theoretical basis of Black Hole. The Black Hole cannot exist, which indicates that the general relativity will not apply to the case of an object being in motion at the high velocity. Thus, we can draw a conclusion from the above analysis that the general relativity will apply only when the motion velocity of an object is less than the velocity of light. 


\section{Conclusion}

The above discussion shows that the special relativity has no transformation formula of the basic physical force or the method to judge the operating speed and operation record of any moving object in the actual time. This paper describes what the immutable axiom of the force balance is, and creates the transformation formula of the force accordingly. Based on the experimental results of J.C. sHafele and R.E. Keating, the problem on the operating speed of the time is analyzed, and it is inferred that the time delay has two different natures, i.e. relative time delay and absolute time delay, and both are real delay. The relative time delay refers to the observation result obtained by observing the time variation of the other moving reference system from one reference system; and the absolute time delay refers to the actual time delay of the object in a circular motion, and by virtue of the absolute time delay, we can infer the operating speed and operation record of any moving object in the actual time. For the general relativity, it is thought that the acting force of the acceleration is different from the nature of universal gravitation, because the acting force of the acceleration is energy transfer force, and the universal gravitation is the field force. Based on the axiom of the immutable force balance, it is concluded that the gravitational mass is constant and is irrelevant to the motion; therefore, the equivalence principle is false in principle. Only when the motion speed of the object is very small relative to the light speed can the equivalence principle be approximately true and the analysis of the general relativity be in line with reality; however, when the motion speed of the object is relatively big, the gravitational mass has obvious difference with the inertial mass, the equivalence principle is false, and the inference of the general relativity is not correct. Taking the black hole for example: the black hole would not be there if the gravitational mass is constant; considering the analysis of the structure of matter, it is inferred that the black hole cannot be the real material composed of atoms, thus indicating that the inference made by the general relativity about the high-speed motion of the object is not in line with reality.

\section{References}

Black Hole. (2014). In Baidu.com.

Tsinghua University electrodynamics teaching and research group (1978). Electrodynamics compiled by Tsinghua University electrodynamics teaching and research group. China Higher Education Press. (1978)

Wu, S. B. (1987). Basics of Theory of Relativity. Shaanxi Science \& Technology Press.

Yao, K. X. (2013). Set up invariable axiom of force equilibrium and solve problems about transformation of force and gravitational mass. Applied Physics Research, 5(1), 72.

Yao, K. X. (2015). A New Explanation of Deflection Results of Charged Particles in High-velocity Motion in Magnetic Field - Correction of Lorentz Force. Applied Physics Research, 7(5), 13-21.

Yao, K. X. (2015). Explanation of Electromagnetics by Motion of Electric Field LAP LAMBERT. Academic Publishing.

\section{Copyrights}

Copyright for this article is retained by the author(s), with first publication rights granted to the journal.

This is an open-access article distributed under the terms and conditions of the Creative Commons Attribution license (http://creativecommons.org/licenses/by/3.0/). 